\title{
Von der Sonderregelung zur Beschäftigungsnorm: Minijobs im deutschen Sozialstaat
}

Minijobs sind in ihrer Bedeutung nicht auf Beschäftigungsverhältnisse für „hinzuverdienende“ Ehefrauen zu reduzieren, sondern Beschäftigte im Minijob sind eine heterogene Erwerbsgruppe. Durch die vielfache Bezugnahme des Sozialrechts auf die $400 €$-Grenze - insbesondere hinsichtlich der Familienkrankenversicherung, des Steuerrechts und der Hinzuverdienstregelungen für Rentnerinnen und Rentner, Arbeitslose und Studierende - haben sich Minijobs vom sozialrechtlichen Sonderfall zu einer Beschäftigungsnorm für unterschiedliche Erwerbsgruppen entwickelt. Lassen sich diese Gruppen quantifizieren? Welche Anreizsysteme beinhaltet das Sozialrecht? Unterscheiden sich die Auswirkungen auf die soziale Absicherung der Betroffenen und welche Konsequenzen ergeben sich für die Finanzierung der Sozialversicherungsträger?

GERHARD BÄCKER, STEFANIE NEUFFER

\section{Problemstellung}

Die als Minijobs bezeichneten geringfügigen Beschäftigungsverhältnisse beruhen auf sozialversicherungs- und steuerrechtlichen Ausnahmeregelungen: Seit der Reform im Jahr 2003 gilt, dass für geringfügige Haupt- und Nebenbeschäftigungen bis zu einer Verdienstgrenze von $400 €$ im Monat bzw. $4.800 €$ im Jahr, aber ohne Begrenzung der Arbeitszeit, keine Arbeitnehmerabgaben entrichtet werden müssen. ${ }^{0}$ Ebenfalls beitragsfrei bleiben kurzfristige Beschäftigungen, also auf zwei Monate oder auf $50 \mathrm{Ar}$ beitstage im Kalenderjahr begrenzte, nicht berufsmäßige Beschäftigungen ohne Verdienstobergrenze. Im Gegenzug entstehen aufgrund der Versicherungsfreiheit auch keine sozialversicherungsrechtlichen Ansprüche. ${ }^{2}$

In diesem Beitrag stehen die Auswirkungen auf das Arbeitsangebot im Mittelpunkt der Analyse, denn die beitragsrechtliche Sonderstellung konstituiert ein spezifisches Arbeitsangebot einzelner Beschäftigtengruppen: Sie schafft monetäre Anreize, das Arbeitsangebot auf wenige Arbeitsstunden bzw. auf ein niedriges Entgelt zu begrenzen. Ziel des Beitrages ist, die Beschäftigtengruppen, auf die diese Regelungen abstellen, auszudifferenzieren und zu quantifizieren. Denn die geringfügig Beschäftigten sind keinesfalls eine homogene Gruppe, sondern unterscheiden sich in ihrem sozialen und sozialrechtlichen Status erheblich. Um diese Unterschiede zu verstehen, werden die sozialrechtli- chen Rahmenbedingungen näher ausgeleuchtet und die Auswirkungen auf die soziale Absicherung der Betroffenen wie auch auf die Finanzen der Sozialversicherungsträger dargestellt.

\section{Sozialversicherungsrechtliche Sonderregelungen}

\subsection{Beschäftigungsformen und Risiken}

Da das lohnzentrierte System der deutschen Sozialversicherung den Schutz vor den Standardrisiken des Lebens unmittelbar an ein Beschäftigungsverhältnis und eine entsprechende Beitragszahlung bindet, führt die bei der

\footnotetext{
(1) Die Arbeitgeber zahlen hingegen Pauschalbeiträge von $15 \%$ zur Gesetzlichen Rentenversicherung (GRV) und $13 \%$ zur Gesetzlichen Krankenversicherung (GKV), eine Pauschalsteuer von $2 \%$ und Sonderumlagen von $0,74 \%$. In der Summe wird damit der reguläre Arbeitgeberbeitragssatz von etwa $20 \%$ merklich überschritten. Deutlich geringere Sätze fallen an, wenn Privathaushalte die Arbeitgeber sind.
}

(2) Die Arbeitgeberbeiträge zur GRV begründen jedoch geringe Ansprüche. 
geringfügigen Beschäftigung eingeräumte Abweichung von der Versicherungspflicht zu dem grundlegenden Problem, dass sowohl bei zukünftigen Lebensereignissen (wie beispielsweise bei Erwerbsminderung oder im Alter) als auch bei aktuellen Risiken (wie bei Krankheit oder Arbeitslosigkeit) eine Sicherungslücke eintritt. Vor allem die fehlende Absicherung im Krankheitsfall wird im Minijob zu einem Problem, da das Risiko, zu erkranken und gegebenenfalls mit hohen Kosten konfrontiert zu werden, gleichsam alltäglich ist und somit kurzfristig eintreten kann. Hingegen lässt sich die fehlende Absicherung bei den Risiken wie Alter und Erwerbsminderung von den Betroffenen minder gewichten, weil mit diesem Risikoeintritt in aller Regel erst in fernerer Zukunft gerechnet werden muss. Zudem orientiert sich die Höhe von Alters- und Erwerbsminderungsrenten am gesamten Erwerbsverlauf und -einkommen. Die Auswirkungen eines fehlenden Anwartschaftsaufbaus hängen damit entscheidend davon $a b$, wie lange die geringfügige Beschäftigung in der Erwerbsund Versicherungsbiografie andauert. Eine nur zwischenzeitliche versicherungsfreie Beschäftigung hat insofern eine grundlegend andere Bedeutung als ein längerfristiger Verbleib in diesem Arbeitsverhältnis.

Die Aufnahme einer geringfügigen Beschäftigung ist nur bei jenen Personen denkbar, die bereits anderweitig materiell und sozial abgesichert, insbesondere krankenversichert sind. ${ }^{3}$ Hier greift das zweite zentrale Merkmal des deutschen Sozialversicherungssystems: Die abgeleitete Absicherung über den Familien- und Eheverbund sowie die Vernetzung zwischen den Sozialversicherungszweigen. Während bei den geringfügig Nebenbeschäftigten die in der Hauptbeschäftigung geltende Krankenversicherung (pflichtig bis zur Versicherungspflichtgrenze) greift, werden bei den geringfügig Hauptbeschäftigten folgende Regelungen wirksam:

- Ehepartner (und eingetragene Lebenspartner) sind im Rahmen der beitragsfreien Mitversicherung von Familienangehörigen in der Gesetzlichen Krankenversicherung (GKV) versichert;

- Schüler sind ebenfalls durch die Familienversicherung abgesichert;

- Studierende (bis zu einer Altersgrenze von 25 Jahren) unterliegen dem Schutz der Familienversicherung oder der Studentischen Krankenversicherung;

- Rentner sind in der Krankenversicherung der Rentner (KVdR) versichert;

- Arbeitslose bleiben Mitglied ihrer bisherigen Krankenversicherung; soweit sie Arbeitslosengeld I oder Arbeitslosengeld II beziehen, werden die Beiträge von den Leistungsträgern übernommen;

- Hilfebedürftige, aber nicht arbeitslose Leistungsempfänger im Rechtskreis des Sozialgesetzbuch Zweites Buch (SGB II) (sog. Aufstocker) realisieren ihren Krankenversicherungsschutz ebenfalls durch die Beitragszahlung des Leistungsträgers.
Wird der Schwellenwert von $400 €$ Arbeitsentgelt überschritten (Einkommen aus mehreren Minijobs werden zusammengerechnet), unterliegen die Beschäftigten in allen vier Versicherungszweigen der Versicherungs- und Beitragspflicht. ${ }^{\boldsymbol{\Phi}}$ Zugleich entfällt die Steuerfreiheit. Allerdings setzt seit der Einführung der sogenannten Midijobs im Jahr 2003 der Eingangsbeitragssatz für die Beschäftigten nur auf einem reduzierten Niveau von ca. $10 \%$ an, um einen abrupten Sprung in der Beitragsbelastung zu vermeiden. Mit steigendem Bruttoverdienst erhöht sich der Beitragssatz gleitend; bei einem Einkommen von $800 €$ wird dann das reguläre Niveau erreicht. Durch die Beitragsentrichtung in dieser Gleitzone werden in der Rentenversicherung (GRV) und Arbeitslosenversicherung (ALV) Ansprüche erworben, die in ihrer Höhe jedoch den niedrigen Beitragssätzen angepasst sind. In der Gesetzlichen Krankenversicherung (GKV) hingegen besteht entsprechend dem Bedarfs- und Sachleistungsprinzip ein voller Leistungsanspruch einschließlich Krankengeld.

Wird bei einer geringfügigen Nebenbeschäftigung die Schwelle von $400 €$ überschritten, werden die Einkommen aus Haupt- und Nebenbeschäftigung zusammengerechnet und regulär verbeitragt (jedoch ohne Beiträge zur Arbeitslosenversicherung für den Nebenjob). Allerdings: Bei zwei Nebenjobs von jeweils bis zu $400 €$ bleibt ein Nebenjob (und zwar der zuerst aufgenommene) abgabenfrei.

Dieses komplexe Regelungsgeflecht lässt - zumindest theoretisch - eine breite Vielfalt von Beschäftigungsformen $\mathrm{zu}^{\boldsymbol{\Theta}}{ }^{\boldsymbol{\omega}}$ in denen keine oder nur niedrige Arbeitnehmerbeiträge entrichtet werden müssen. Midijobs oder Kombinationen mit einem Midijob erweisen sich dabei für alle diejenigen als besonders vorteilhaft, die - wie Selbstständige und ihre Angehörigen - ansonsten keinen Zugang zur GKV haben und in der PKV hohe risikobezogene Individualprämien entrichten müssen. Hingegen fällt zu Beginn der MidiZone der Arbeitnehmerbeitrag zur GKV nur sehr gering aus: Bereits mit einer Beitragszahlung von etwa $17 €$, die bei einem Verdienst von $401 €$ anfällt, steht das gesamte Leistungsspektrum der GKV zur Verfügung. Wer auf einen Krankenversicherungsschutz durch ein Beschäftigungsverhältnis nicht angewiesen ist, weil bereits eine alternative

(3) Seit 2009 besteht eine Krankenversicherungspflicht für die gesamte Bevölkerung. Eine freiwillige Versicherung in der GKV oder eine private Versicherung kommt bei einem Minijob kaum in Betracht, da die Höhe der Beiträge bzw. Prämien in keinem Verhältnis zu dem Maximaleinkommen von $400 €$ steht.

(4) Für kurzfristig Beschäftigte gilt keine Verdienstobergrenze, selbst ein hohes Einkommen führt nicht zur Versicherungsund Beitragspflicht. Dies gilt auch dann, wenn ein Minijob mit einer kurzfristigen Beschäftigung zusammentrifft; beide Einkommen werden nicht zusammengerechnet.

5 Ob diese Beschäftigungsformen tatsächlich entstehen, hängt - nicht zuletzt aufgrund der Machtverhältnisse am Arbeitsmarkt - entscheidend von der Arbeitsnachfrage ab. 
Absicherung besteht, und auch eine eigenständige Sicherung im Alter oder bei Erwerbsminderung als nicht wichtig erachtet, ist hingegen daran interessiert, den Grenzwert von $400 €$ der Minijobs nicht zu überschreiten. Der fixe Grenzwert kann deshalb auch dazu verleiten, ein übersteigendes Einkommen nicht anzugeben bzw. zu verschleiern.

\subsection{Auswirkungen auf die Finanzierung der Sozialversicherung}

Die Beitragsfreiheit der Arbeitnehmer in der Minijob-Zone wirkt sich auf die Haushalte der Sozialversicherungszweige unterschiedlich aus. So kommt es in der Renten- wie auch in der Arbeitslosenversicherung kaum zu größeren Finanzierungsproblemen, da aus den beitragsfreien Entgelten auch keine Ansprüche erwachsen. ${ }^{\bullet}$ Anders sieht es in der Kranken- und Pflegeversicherung aus, denn trotz der Beitragsfreiheit bestehen in der Regel volle abgeleitete Leistungsansprüche. Zwar zahlen die Arbeitgeber einen Pauschalbeitrag von $13 \%$, der jedoch unter dem regulären GKV-Gesamtbeitragssatz liegt. Der Sozialen Pflegeversicherung (SPV) fließen überhaupt keine Beitragseinnahmen zu. Will man die Höhe der Einnahmeverluste abschätzen, muss geklärt werden, ob und in welchem Maße Mini-Jobs Verdrängungseffekte zulasten sozialversicherungspflichtiger Beschäftigung auslösen und deswegen auf reguläre Beitragseinnahmen verzichtet werden muss (vgl. Bäcker 2007, S. 108ff.). Zwei Extreme können die Spannweite der möglichen fiskalischen Folgen verdeutlichen:

Unterstellt man, dass ohne die Minijob-Regelung das Arbeitsangebot und die entsprechende Arbeitsnachfrage erst gar nicht entstanden wären, dass es sich also hierbei um zusätzliche Beschäftigung handelt, dann würden bei der GKV infolge des Arbeitgeberpauschalbeitrags erhebliche Mehreinnahmen anfallen. Das gleiche träfe zu, wenn Schwarzarbeit über diesen Weg eingedämmt würde. Ein exakt gegenteiliger Effekt ist zu erwarten, wenn entweder die Ausbreitung von Minijobs mit einer Verdrängung von vorhandener sozialversicherungspflichtiger Beschäftigung einhergeht oder wenn neue Arbeitsplätze geringfügig und nicht versicherungspflichtig geschaffen werden. ${ }^{\circ}$ Dann verringern sich die Einnahmen von GKV und SPV bzw. den Versicherungsträgern entgehen zusätzliche Einnahmen.

Die theoretisch maximale Belastung ergibt sich dabei bei einem Verdrängungseffekt von $100 \%$ : Wenn statt eines Beitragssatzes von $17,45 \%$ (GKV inkl. Sonderbeitrag + SPV inkl. Kinderlosenbeitrag) nur der Pauschalbeitrag von $13 \%$ abgeführt wird, errechnet sich bei einem Minijob-Monatseinkommen von im Schnitt $283 €$ (Statistisches Bundesamt 2011a) je Fall eine Mindereinnahme von $151 €$ im Jahr, was bei 7,4 Mio. Minijobs einer Summe von etwa 1,1 Mrd. € entspricht - bei Gesamteinnahmen von GKV (Gesundheitsfonds) und SPV in Höhe von gut $170 \mathrm{Mrd}$. $€$. Die Belastung der Sozialversicherungsträger bleibt also selbst unter der extremen Annahme einer vollständigen Substi- tution überschaubar. Fällt indes, was zu erwarten ist, der Verdrängungseffekt niedriger aus, müssen neben den Mindereinnahmen auch die Mehreinnahmen berücksichtigt werden. Schon bei einem Verdrängungseffekt von $75 \%$ kommt es nahezu zu einem Ausgleich: Den Mindereinnahmen von etwa $0,84 \mathrm{Mrd}$. $€$ stehen dann Mehreinnahmen von 0,82 Mrd. $€$ gegenüber $(283 € \times 12$ x $13 \%$ Pauschalbeitrag x 1,85 Mio. Minijobs).

\section{Struktur der Beschäftigtengruppen in Minijobs}

Die Datenlage über Ausmaß, Dynamik und Struktur der Beschäftigtengruppen im Bereich der Mini- und auch Midijobs erweist sich als unzureichend. Differenzierte Aussagen lassen sich kaum treffen. Zwar stehen mit der laufenden Statistik der Bundesagentur für Arbeit (BA) und den vierteljährlichen Qualitätsberichten der Minijobzentrale gleich zwei Datenquellen zur Verfügung. Allerdings zeigen sich z.T. erhebliche Abweichungen hinsichtlich Niveau und Struktur der Beschäftigten, was vor allem daran liegt, dass die auf dem gemeinsamen Meldeverfahren zur Sozialversicherung basierende BA-Beschäftigtenstatistik ihre Ergebnisse erst mit sechs Monaten Wartezeit veröffentlicht und damit auch verspätete An- und Abmeldungen erfasst (BA 2011a). Sie ist insofern aussagekräftiger als die Qualitätsberichte der Minijob-Zentrale. Allerdings bietet die Beschäftigtenstatistik nur wenige Informationen über die sozio-demografischen Merkmale einzelner Beschäftigtengruppen. Hier lässt der Mikrozensus differenziertere Aussagen zu, dieser unterzeichnet jedoch die Zahl der geringfügig Beschäftigten, da den Befragten die sozialrechtlichen Regelungen nicht immer klar sind oder die Befragten ihren Hauptstatus beispielsweise als Hausfrauen, Rentner oder Studierende definieren (vgl. Körner/Puch 2009, S. 535).

(6) Würde in der GRV statt des Arbeitgeberpauschalbeitrags (15\%) der reguläre Beitragssatz $(19,9 \%)$ gelten, käme es jedoch kurz- und mittelfristig zu einer Entlastung, da die Mehrausgaben infolge der höheren Rentenanwartschaften erst mit einem erheblichen Zeitverzug anfallen.

7 In diese Richtung tendiert nicht nur die Einschätzung des Rheinisch-Westfälischen Instituts für Wirtschaftsforschung (RWI) und des Instituts für Sozialforschung und Gesellschaftspolitik (ISG) im Rahmen der Evaluation der HartzGesetze (RWI/ISG 2006). Auch die Beschäftigtenstruktur in einzelnen Branchen, so Handel, Gebäudereinigung und Gastgewerbe, in denen im Durchschnitt $40 \%$ aller Beschäftigungsverhältnisse Minijobs sind (vgl. Voss/Weinkopf in diesem Heft), weist darauf hin, dass es sich hier nicht etwa um verzichtbare, sondern für den Betriebsablauf unabding bare Beschäftigungsverhältnisse handelt, die, wenn es die Minijobs nicht gäbe, von den Unternehmen sozialversicherungspflichtig eingerichtet würden. 
Bekannt sind die Daten über Niveau und Entwicklung der geringfügigen Haupt- und Nebenbeschäftigten insgesamt (vgl. BA 2011b): Zwischen 2003 und 2010 hat sich die Zahl der geringfügig Hauptbeschäftigten von knapp 4,4 auf gut 4,9 Mio. erhöht, wobei seit 2006 die Zahlen weitgehend konstant sind. Stark zugenommen haben die Nebenjobs - von etwa 1,2 Mio. (2003) auf nahezu 2,5 Mio. (2010). Hier hält der Anstieg, wenn auch verlangsamt, seit Jahren an. Die kurzfristigen Beschäftigungen sind in diesen Werten nicht enthalten: Sie werden für Ende 2010 auf fast 296.000 beziffert. Die Zahl der Midijobs lag Ende 2010 bei rund 1,3 Mio. Von diesen betrafen aber nur etwa 762.000 (57,8\%) die Gleitzone, während die übrigen als „Mischfälle“ eingestuft werden. Hierbei handelt es sich um Arbeitsverhältnisse, bei denen das Arbeitsentgelt im Jahresverlauf teilweise außerhalb der Gleitzone liegt. Zu Beginn der Neueinführung der Gleitzonenregelung haben sich die entsprechenden Beschäftigungsverhältnisse deutlich erhöht, von 143.000 Ende 2003 auf über 625.000 Ende 2006 (BA 2007). Seitdem ist der Zuwachs fast zum Erliegen gekommen.

Die nach wie vor geringe Resonanz der Midijobs weist zugleich darauf hin, dass sich die $400 €$-Grenze gleichsam verselbstständigt und zu einer Art verhaltenssteuernder Beschäftigungsnorm entwickelt hat. Beschäftigte werden dadurch in ein niedriges Arbeitszeitvolumen „eingesperrt“. Der Blick auf die Einkommensentwicklung bestärkt diese Aussage: Seitdem im Jahr 2003 der Grenzwert von $400 €$ festgelegt worden ist, haben sich die nominalen (!) tariflichen Löhne und Gehälter je Arbeitnehmer in diesem Zeitraum um durchschnittlich 15,5\% erhöht (WSI 2011). Wenn man unterstellt, dass sich diese Steigerung zumindest teilweise auch in den Bruttostundenentgelten bei den Minijobs widerspiegelt, müssten bei gegebener Arbeitszeit in den letzten Jahren immer mehr Beschäftigte mit ihrem Einkommen den Wert von $400 €$ überschritten und in den Bereich der Midijobs gewechselt sein. Offensichtlich ist dies nicht der Fall. Anzunehmen ist vielmehr, dass bei Lohnerhöhungen eher die Arbeitsstunden reduziert oder Ausweichverfahren eingeschlagen werden (vgl. Voss/Weinkopf in diesem Heft).

Fragt man nach der Zusammensetzung der geringfügig entlohnten Beschäftigten, liegen eindeutig abgegrenzte Ergebnisse allein für das Jahr 2004 vor: In einer repräsentativen Befragung gaben 30,2\% an, unmittelbar vor Aufnahme des Minijobs ausschließlich Hausarbeit geleistet zu haben, $11,5 \%$ waren arbeitslos gemeldet, $13,1 \%$ Schüler oder Student, 12,4\% Rentner oder Pensionär, $11 \%$ befanden sich in Elternzeit und der Rest war zuvor regulär erwerbstätig (RWI 2004, S. 67). Nun können diese Befunde allerdings nicht auf die Gegenwart übertragen werden, da sie in der Einführungsphase der Neuregelungen erhoben worden sind und sich seitdem die sozioökonomischen Rahmenbedingungen merklich verändert haben.

Da die Beschäftigtenstatistik nicht nach Erwerbs-, Familien- und Ausbildungsstatus, sondern nur nach Alter, Geschlecht und Qualifikation unterscheidet, mögen folgende Größen - bezogen auf Ende 2010 - als Annäherung dienen (BA 2011b):
(1) Unter den ausschließlich geringfügig Beschäftigten sind $18,1 \%$ jünger als 25 Jahre. Hierbei dürfte es sich zu einem großen Teil, wenn nicht überwiegend, um Schüler und Studierende handeln.

(2) Frauen stellen zwei Drittel (66,2\%) der ausschließlich geringfügig Beschäftigten; Frauen im mittleren Lebensalter ( 25 bis 50 Jahre und 50 bis 55 Jahre) sogar mehr als drei Viertel (76,0\% und 77,2\%). Es spricht vieles dafür, dass sich hierunter zu einem erheblichen Anteil verheiratete, hinzuverdienende „Hausfrauen“ finden. Diese Gruppe dürfte auch bei den Beschäftigten in den Privathaushalten dominieren: Die Minijob-Zentrale weist für das 4. Quartal 2010 hier 222.000 Beschäftigte aus, dabei handelt es sich zu $92 \%$ um Frauen, von denen sich 77,7\% in der Altersgruppe zwischen 25 und unter 60 Jahren befinden (Minijob-Zentrale 2010).

(3) Unter den ausschließlich geringfügig Beschäftigten befinden sich 17,1\% in der Altersspanne zwischen 55 und 65 Jahren, darunter werden - neben den hinzuverdienenden Ehefrauen (zu einem allerdings schwer abschätzbaren Teil) - viele Bezieher einer vorgezogenen Alters- oder einer Erwerbsminderungsrente sein. Personen im Alter über 65 Jahre, die die Regelaltersgrenze erreicht haben und nahezu vollständig eine Rente beziehen, machen $10,4 \%$ der geringfügig Beschäftigten aus.

(4) Aus der SGB-II-Statistik schließlich lässt sich entnehmen, dass im Jahr 2010 über 722.000 Leistungsbezieherinnen und -bezieher ein Bruttoeinkommen von bis zu $400 €$ bezogen haben. Das entspricht in etwa 14,6\% aller ausschließlich geringfügig Beschäftigten (BA 2011c). Über die Größenordnung der im Minijob-Bereich erwerbstätigen Bezieher der Versicherungsleistung Arbeitslosengeld liegen hingegen keine Informationen vor.

Vergleicht man dieses Bild im Zeitverlauf seit 2006, so lassen sich keine grundlegenden Verschiebungen erkennen: Unter den geringfügig Hauptbeschäftigten ist der Anteil der Jüngeren leicht gestiegen, der der Älteren leicht gefallen. Der Frauenanteil zeigt sich marginal rückläufig (BA 2007).

Die aufgeführten Querschnittdaten geben allerdings noch keine Antwort auf die entscheidende Frage, mit welcher Dauer in der Erwerbsbiografie ein Minijob von den Beschäftigten ausgeübt wird. Erst wenn beurteilt werden kann, ob sich die versicherungsfreie Beschäftigung als eine eher kurzfristige Episode darstellt oder ob sie über Jahre hinweg praktiziert wird, lässt sich ermessen, welche Auswirkungen dies auf die individuelle Einkommensposition und Aufstiegschancen sowie auf die Höhe einer späteren Rente hat (vgl. dazu auch Klenner/Schmidt in diesem Heft). Einen lediglich begrenzten Hinweis auf die Beschäftigungsdauer liefern die Befunde der Minijob-Zentrale: Danach dauerten $38 \%$ der Minijobs Ende 2010 weniger als ein Jahr an, $21 \%$ bis zu zwei Jahre und $28 \%$ mehr als drei Jahre (vgl. 
ABB. 1

\section{Höhe des Netto-Arbeitsentgelts im Bereich zwischen 0 und $900 €$ brutto in Steuerklasse V}

Angaben in Euro

nettoentgelt $\quad$ Sozialversicherungsbeiträge $\quad$ Lohnsteuer

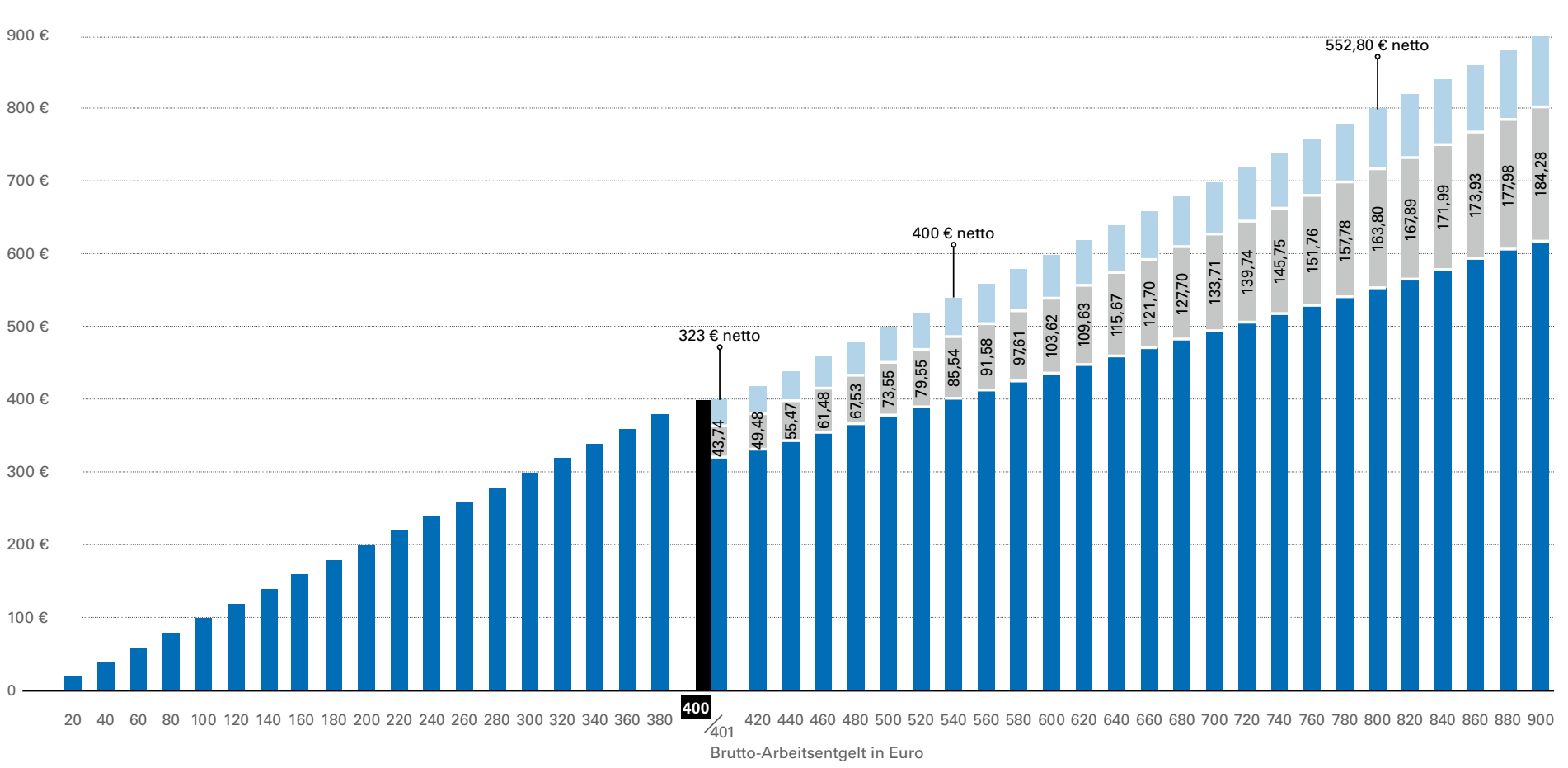

Quelle: Berechnungen der Autoren nach Deutsche Rentenversicherung (2010).

Minijob-Zentrale 2010). Eine unzureichende Aussagekraft haben diese Daten deswegen, weil nur die Dauer ein und desselben Beschäftigungsverhältnisses erfasst wird. Offen bleibt, wie lange eine Person über die Jahre hinweg geringfügig beschäftigt ist, unabhängig davon, welche Unterbrechungen aufgetreten und ob neue Beschäftigungsverhältnisse eingegangen worden sind.

\section{Spezifische Bedingungen einzelner Beschäftigtengruppen: Auswirkungen auf Arbeitsanreize und Alterssicherung}

Für geringfügig Beschäftigte ist von entscheidender Bedeutung, ob eine Tätigkeit in einem Minijob am Beginn oder am Ende einer regulären und kontinuierlichen Erwerbsphase steht, ob sie dazu dient, in Zeiten von Arbeitslosigkeit oder Kindererziehung die Transferzahlungen aufzustocken, oder ob Minijobs ein gleichsam „normaler“
Bestandteil in der Mitte der Erwerbsbiografie sind. Im Folgenden werden Beschäftigtengruppen näher analysiert, die sich mit Blick auf ihren sozialrechtlichen Status unterscheiden.

\subsection{Ehefrauen}

Quasi zur Normalität haben sich geringfügige Beschäftigungsverhältnisse im Erwerbsleben einer großen Gruppe von (westdeutschen) Ehefrauen entwickelt, deren Erwerbseinkommen nach dem Modell der sogenannten modifizierten Versorgerehe eher einen „Zuverdienst“ darstellt und deren soziale Sicherung im Wesentlichen abgeleitet über den Ehemann erfolgt - im Alter über die Rente des Ehemannes bzw. die Hinterbliebenenversorgung und im Krankheitsfall über die Familienversicherung der GKV. ${ }^{\boldsymbol{0}}$

8 Die beitragsfreie Mitversicherung von Ehefrauen erfolgt unabhängig davon, ob Kinder versorgt werden. In der Kombination mit der Beitragsbemessungsgrenze (BMG) erweist sich die Mitversicherung vor allem dann von Vorteil, wenn die Männer gut verdienen. 
Die Ausgestaltung der Minijobs trägt dazu bei, dass dieses geschlechtshierarchische Erwerbsmodell gefördert und festgeschrieben wird. Die Anreize, sich auf eine Erwerbsbeteiligung in diesem Segment des Arbeitsmarktes einzulassen und dies nicht nur als kurzfristige Überbrückungsund Übergangsphase zu verstehen, werden in besonderem Maße offensichtlich, wenn auch steuerrechtliche Aspekte berücksichtigt werden. Infolge des Ehegattensteuersplittings und der üblichen Steuerklassenwahl (Kombination von III und V) bei einem zwischen den Ehepartnern stark abweichenden Einkommen erweist sich aus Sicht des Paares ein Überschreiten der $400 €$-Grenze der Frau als unattraktiv, denn trotz der Einführung der Gleitzone ${ }^{\bullet}$ errechnet sich immer noch ein erheblicher Nettoeinkommensverlust (Abbildung 1). So liegt bei einem Einkommen von $401 € \mathrm{der}$ Nettobetrag nur noch bei $323 €$, und erst bei einem Einkommen von $540 €$ werden wieder $400 €$ netto erreicht. Werden bei einem gegebenen Stundenlohn die Arbeitszeit und das Bruttoeinkommen von $400 €$ auf $800 €$ verdoppelt, so erhöht sich das Nettoentgelt nur um 38,2\% - von $400 €$ auf 552,80€. Die hohen Belastungen in der Steuerklasse V gleichen sich in der gesamten Steuerbelastung des Ehepaares zwar aus, aber im Ergebnis wird die Entscheidung über Art und Ausmaß der Erwerbsbeteiligung von Ehefrauen stark durch die monetären Anreizeffekte des sozial- und steuerrechtlichen Sonderstatus von Minijobs beeinflusst.

Daraus erwächst das Problem, dass die Frauen bei längerer Dauer einer nicht versicherungspflichtigen Beschäftigung keine ausreichende eigenständige Alterssicherung aufbauen. Zwar kann dem mit dem Argument entgegengetreten werden, dass die Rentenanwartschaften bei einem Einkommen von bis zu $400 €$ auch dann minimal bleiben, wenn volle Beiträge entrichtet werden. Tatsächlich würden in einem Jahr bei einem monatlichen Verdienst von durchgehend $400 €$ Rentenansprüche von lediglich 4,30 € (2011) erworben. Diese geringe Betrag mag auch ein zentraler Grund dafür sein, dass im Juni 2011 lediglich 5,1\% der Minijobber im gewerblichen Bereich auf die Versicherungsfreiheit verzichten (im Juni 2006 waren es 2,6\%) und den Gesamtbeitragssatz durch Zahlung eines Aufstockungsbeitrags von 4,9\% auf das reguläre Niveau von 19,9\% anheben (Minijob-Zentrale 2011). Vernachlässigt wird dabei jedoch, dass nur bei regulärer Beitragszahlung „vollwertige“ Leistungsansprüche entstehen. Dies bedeutet, dass jeder Beschäftigungsmonat in vollem Umfang für die Rente zählt. Damit können alle Wartezeiten, zum Beispiel für vorgezogene Altersrenten, erfüllt sowie Ansprüche auf Leistungen der Rehabilitation und auf eine Rente wegen Erwerbsminderung erworben werden.

Entscheidender ist aber der Verweis auf die mittelbaren Wirkungen der $400 €$-Grenze: Als Normgröße für Frauenbeschäftigung subventioniert, etabliert und (in Westdeutschland) kulturell verfestigt, verhindert sie, dass das Arbeitsangebot ausgeweitet und der eigenständige Alterssicherungsanspruch über ein höheres Stundenvolumen und ein höheres Einkommen verbessert wird.

\subsection{Arbeitslose und erwerbstätige Leistungsberechtigte}

In einer grundlegend anderen Situation stehen Arbeitslose. Wenn Arbeitslose einen Nebenverdienst aufnehmen - ohne ihren Arbeitslosenstatus, ihre Leistungsansprüche und den über die Leistungsträger sichergestellten Krankenversicherungsschutz gefährden zu wollen -, dann steht dahinter die Absicht, das Einkommen aufzubessern, womöglich Kontakte zur Arbeitswelt zu knüpfen und die Reintegrationschancen zu verbessern (vgl. Dietz et al. 2009).

Hinsichtlich der Regelungen ist dabei zwischen den Rechtskreisen des SGB III und SGB II zu unterscheiden: Wer 15 Wochenstunden und mehr arbeitet, ist nach dem SGB III nicht mehr arbeitslos und verliert seinen Anspruch auf die Versicherungsleistung Arbeitslosengeld (ALG) I. Wird unterhalb dieses Stundenvolumens neben dem Bezug von ALG I eine Nebentätigkeit aufgenommen, wird das ALG I um das erzielte (Netto-)Einkommen gekürzt. Die Höhe des Nebeneinkommens kann dabei zwar die Geringfügigkeitsgrenze von $400 €$ übersteigen. Üblich dürfte dies jedoch nicht sein, denn stets bleibt nur ein maximaler Freibetrag in Höhe von $165 €$ anrechnungsfrei. Eine Nebentätigkeit mit einem höheren Einkommen verbessert also das Gesamteinkommen nicht.

Beim SGB II folgt aus dem Nachrangigkeits- und Bedürftigkeitsprinzip, dass ein Nebeneinkommen auf die Leistungsansprüche angerechnet wird. Im Unterschied zum SGB III können Personen, die nicht arbeitslos, sondern erwerbstätig sind, Leistungen beziehen. Bezugsvoraussetzung sind Erwerbsfähigkeit und der Status der Hilfebedürftigkeit im Kontext der Bedarfsgemeinschaft. Ein kompliziertes, seit Verabschiedung des SGB II mehrfach geändertes System eines absoluten Freibetrages und relativer Freibeträge oberhalb dessen soll einerseits Anreize zur Aufnahme von Erwerbsarbeit setzen und andererseits verhindern, dass sich der Kombilohn-Charakter des SGB II weiter ausdehnt. Der absolute Freibetrag liegt bei $100 €$, in den Verdienstbereichen zwischen $100 €$ und $1.000 €$ bleiben weitere $20 \%$ sowie zwischen $1.000 €$ und $1.200 €$ (bei Paaren mit unterhaltsberechtigten Kindern: $1.500 €$ ) weitere $10 \%$ anrechnungsfrei. Bei einem $400 €$-Beschäftigungsverhältnis bleiben $160 €$ übrig und erhöhen das verfügbare Gesamteinkommen der Bedarfsgemeinschaft. Im Unterschied zum SGB III lohnt es sich aber durchaus, ein höheres Einkommen zu erzielen, da der Anspruch auf Arbeitslosengeld II nicht an den Status der Arbeitslosigkeit gebunden ist und insofern das Gesamteinkommen, wenn auch nur langsam, steigt. ${ }^{\circledR}$ Zu berücksichtigen ist dabei, dass die anrechnungsfreien Beträge nicht nur für Leistungsberechtigte gelten, die aus der Arbeitslosigkeit heraus eine Arbeit aufnehmen; sie gelten für alle

( Vor Einführung der Gleitzone fielen die Einkommens sprünge beim Überschreiten der Geringfügigkeitsgrenze noch steiler aus (vgl. Koch/Bäcker 2003, S. 97). 
Erwerbstätigen, die aufgrund ihres niedrigen Einkommens (bzw. hohen Bedarfs) hilfebedürftig sind. Insofern handelt es sich beim ALG II immer auch um eine bedürftigkeitsgeprüfte Lohnaufstockung. Die oben skizzierten Daten weisen gleichwohl darauf hin, dass von den gut 1,3 Mio. Leistungsbeziehern in abhängiger Beschäftigung mehr als die Hälfte $(57,1 \%)$ in einem Minijob arbeitet, davon zu einem Teil auch nur bis zu einem Einkommen von bis zu $200 €$, um den absoluten Freibetrag von $100 €$ auszuschöpfen. In dieser Gruppe finden sich viele nichtarbeitslose Alleinerziehende, die wegen der Erziehungsverpflichtungen dem Arbeitsmarkt nicht zur Verfügung stehen und denen ein sozialversicherungspflichtiges Beschäftigungsverhältnis deshalb nicht zugemutet werden kann (u.a. wegen fehlender Kinderbetreuungseinrichtungen) oder die eine solche Tätigkeit nicht finden oder anstreben und deshalb hilfebedürftig bleiben. ${ }^{(1)}$

Für Empfänger von Leistungen des SGB III und des SGB II gilt, dass in Minijobs keine ausreichenden Rentenansprüche erworben werden können. Aber selbst wenn die Versicherungsfreiheit aufgehoben würde: Für die Alterssicherung von Arbeitslosen zu sorgen, bleibt vorrangige Aufgabe des zuständigen Leistungssystems. Besonders im SGB II klaffen hier gravierende Lücken auf. Bis Ende 2010 wurden vom Bund Mini-Beiträge gezahlt (denen 2009 nach zwölf Monaten Arbeitslosigkeit ein Anspruch auf 2,19 € Rente im Monat entsprach). Ab 2011 sind diese Beiträge im Rahmen der Sparmaßnahmen ersatzlos entfallen, die Zeiten werden seitdem als Anrechnungszeiten berücksichtigt. Schon jetzt ist deshalb absehbar, dass für Langzeitarbeitslose die Altersarmut zu einem zentralen Problem werden wird (vgl. Bäcker 2011, S. 175).

\subsection{Rentner}

Hinsichtlich der möglichen Erwerbstätigkeit sind zwei Gruppen von Rentnerinnen und Rentnern zu unterscheiden: Diejenigen, die ihre Altersrente nach Erreichen der Regelaltersgrenze beziehen, können unbeschadet von ihrer Rentenzahlung eine Erwerbstätigkeit ohne Einkommensbegrenzung aufnehmen. Für diejenigen, die eine vorgezogene, in aller Regel mit Abschlägen belegte Altersrente erhalten oder Empfänger einer vollen Erwerbsminde- rungsrente sind, gelten Hinzuverdienstgrenzen in Höhe von $400 €$. Besondere Grenzen gelten für die Rente wegen teilweiser Erwerbsminderung.

Obgleich eine Erwerbstätigkeit über das 65. Lebensjahr hinaus unbegrenzt möglich ist, befindet sich der weit überwiegende Teil der erwerbstätigen Rentnerinnen und Rentner in diesem Alter in einem geringfügigen Beschäftigungsverhältnis: Ende 2010 waren dies ca. 769.000 Personen - gegenüber 126.000 in einem sozialversicherungspflichtigen Beschäftigungsverhältnis (BA 2011b). Das überrascht wenig, da trotz einer Sonderregelung für erwerbstätige Rentner ${ }^{\text {(3) }}$ die gänzliche Beitrags- und Steuerfreiheit der Minijobs attraktiver ist. Dies trifft insbesondere dann zu, wenn davon auszugehen ist, dass im Rentenalter eine Beschäftigung in Vollzeit oder langer Teilzeit eher selten sein dürfte.

Die Größenordnung der Beschäftigung von Alters- und Erwerbsminderungsrentnern im Rahmen der Hinzuverdienstgrenze lässt sich nicht genau ermitteln, weil die ausschließlich geringfügig Beschäftigten im rentennahen Alter $\mathrm{zu}$ großen Teilen auch arbeitslos sein oder zu der Gruppe der „hinzuverdienenden“ Ehefrauen zählen können. Überschreiten Rentner die Hinzuverdienstgrenze ${ }^{\circledR}$ mindert sich automatisch und recht abrupt die Rente, sie wird von einer Voll- in eine Zweidrittel-Teilrente umgewandelt. Durch die gleichzeitig entstehende Beitragspflicht werden aber auch zusätzliche Entgeltpunkte erworben.

Aktuell wird diskutiert, die Hinzuverdienstgrenzen deutlich anzuheben, um es den Beziehern von vorgezogenen Altersrenten zu ermöglichen, ihre niedrigen Renten, vermindert noch um Abschläge, durch eine ausgeweitete Nebentätigkeit aufzubessern. Schon derzeit ist davon auszugehen, dass die Nebenbeschäftigung von Rentnern zu einem Teil durch materielle Zwänge bedingt ist. Der Vorschlag eines erweiterten Hinzuverdienstes mag deshalb aus Sicht der Betroffenen auf Zustimmung stoßen; er würde aber den Charakter der Rentenversicherung grundlegend ändern. Denn das Ziel, nach einem langen Arbeitsleben eine existenz- und lebensstandardsichernde Rente zu garantieren, würde aufgegeben. Mit dem Hinweis auf Zuverdienstmöglichkeiten würden letztlich Armutsrenten legitimiert. Analog zu Kombilohnmodellen würde sich das Arbeitsangebot von Rentnerinnen und Rentnern er-
(1) Erwerbsfähige Hilfebedürftige, die dem Arbeitsmarkt zur Verfügung stehen, können allerdings nicht "frei" entscheiden, ob sie ihr Arbeitsangebot auf 15 Stunden und $400 €$ beschränken oder auf einen Arbeitsplatz mit einem höheren Einkommen und Stundenvolumen wechseln. Da es Ziel des SGB II ist, die Hilfebedürftigkeit zu überwinden, müssen Arbeitsverhältnisse mit einem höheren Einkommen -

soweit vorhanden bzw. im Vermittlungsprozess angeboten angenommen werden. Im Verweigerungsfall drohen Sanktionen.

(1) Im Jahr 2009 ging fast ein Drittel der 636.000 erwerbsfähigen hilfebedürftigen Alleinerziehenden einer Erwerbsarbeit nach, davon etwa zur Hälfte im Bereich einer geringfügigen Beschäftigung (eigene Berechnungen nach BA 2010).
(12) Bei Einkommen über $400 €$ werden erstens keine Beiträge zur Renten- und Arbeitslosenversicherung fällig, denn Arbeitnehmer, die die Regelaltersgrenze überschritten haben, können nicht arbeitslos sein. Es können auch keine rentensteigernden Entgeltpunkte mehr erworben werden, da die Rente endgültig festgesetzt worden ist. Insofern entfällt auch die Beitragspflicht zur Rentenversicherung. Zweitens werden wegen des fehlenden Anspruchs auf Krankengeld nur ein ermäßigter GKV-Beitrag von 7,6\% sowie Beiträge zur SPV erhoben. Die Arbeitgeber müssen hingegen den regulären Gesamtsozialversicherungsbeitrag leisten.

(13 Allerdings darf die Hinzuverdienstgrenze bis zu zweimal im Jahr um $400 €$ überschritten werden. 
höhen - mit der Bereitschaft auch zur Annahme von prekärer Beschäftigung. Unbeantwortet bleibt dabei vor allem die Frage, wie das Einkommen im Alter gesichert werden soll, wenn eine ausgeweitete Nebenerwerbstätigkeit aus gesundheitlichen Gründen nicht mehr möglich ist.

\subsection{Schüler und Studierende}

Im Wintersemester 2010/11 wurden an den deutschen Hochschulen gut 2,2 Mio. Studierende gezählt (Statistisches Bundesamt 2011b). Die Zahlen steigen kontinuierlich an, zehn Jahre zuvor waren es noch 1,8 Mio. Die Gruppe der Schülerinnen und Schüler in der Sekundarstufe II in allgemeinbildenden Schulen, die in der Regel 16 Jahre und älter sind, beziffert die Statistik im Schuljahr 2009/10 auf etwa 1 Mio. (Statistisches Bundesamt 2011c). Es ist offensichtlich, dass es in dieser großen Gesamtgruppe von über 3 Mio. Personen eine hohe Erwerbsbereitschaft und Erwerbsbeteiligung gibt - wegen der gleichzeitigen Beanspruchung durch Schule und Studium allerdings im unteren Stundenspektrum oder nur sporadisch, so in den Schul- und Semesterferien. Insgesamt lassen die Befunde des Mikrozensus (einschließlich Nachbefragung) erkennen, dass sich die traditionelle Phaseneinteilung des Lebensverlaufs, die einen klaren Schnitt zwischen der Ausbildungs- und der Erwerbsphase vorsah, weitgehend verwischt. So weisen Schüler im Jahr 2008 eine Erwerbstätigenquote von $31 \%$ auf, die Studierenden sogar von 50 \% (Körner/Puch 2009, S. 538). In eine vergleichbare Richtung weisen die Befunde aus der 19. Sozialerhebung des Deutschen Studentenwerks (2010, S. 193): Im Jahr 2009 erzielten $65 \%$ der Studierenden einen Verdienst während des Studiums mit einem durchschnittlichen Betrag von $323 €$.

Erwerbstätigkeit wird in der Ausbildungsphase als zwischenzeitlicher Job verstanden, der mit dem Abschluss durch eine qualifikationsadäquate und sozialversicherungspflichtige Beschäftigung abgelöst wird. Ob dies immer zur Zufriedenheit gelingt, bleibt freilich offen. Aber während des „Jobbens" sind die Bereitschaft sowie der Druck groß, auch Arbeiten zu schlechten Bedingungen anzunehmen. Die Arbeit erfolgt vermutlich weitgehend im Rahmen der ausschließlichen geringfügigen Beschäftigung und/oder der kurzfristigen Beschäftigung. Hinzu kommt als Sonderregelung für Studierende der Status des sogenannten Werkstudenten. Daten über die Verbreitung dieser Beschäftigungsform liegen überraschenderweise nicht vor. Als Werkstudent gilt, wer mit allen seinen $\mathrm{Ne}$ benjobs eine regelmäßige Wochenarbeitszeit von insgesamt 20 Stunden nicht überschreitet. ${ }^{\text {() }}$ Dieser Status befreit unabhängig von der Höhe des erzielten Einkommens von Arbeitnehmerbeiträgen zur Arbeitslosenversicherung wie zur Kranken- und Pflegeversicherung: ${ }^{\circledR}$ Lediglich zur Rentenversicherung werden Arbeitnehmerbeiträge fällig. Damit lassen sich die gravierenden Verschlechterungen bei der Anrechnung und Bewertung von Ausbildungszeiten in der Rentenversicherung, die in den zurückliegenden
Jahren immer weiter verschärft worden sind, allerdings nur sehr begrenzt ausgleichen.

\section{Fazit und Ausblick}

Minijobs sind in ihrer Bedeutung nicht auf Beschäftigungsverhältnisse für „hinzuverdienende“ Ehefrauen zu reduzieren. Mittlerweile arbeitet ein breiter Kreis von Personen in diesen Beschäftigungsverhältnissen. Was einst ein randständiger Sonderfall im deutschen Sozialversicherungssystem war, ist heute für mehrere, sich stark unterscheidende Beschäftigtengruppen, die über andere Wege - zumindest kurzfristig - sozial abgesichert sind, zur Orientierungsgröße für individuelle und haushaltsbezogene Entscheidungen der Erwerbsbeteiligung geworden.

Bei der Reformdebatte sind also nicht nur sehr unterschiedliche Lebens- und Interessenlagen der einzelnen Gruppen, sondern auch die jeweiligen Rechtskreise zu berücksichtigen. So kann bei einer Neuregelung, die auf eine Absenkung oder gar Abschaffung der Geringfügigkeitsschwelle und damit auf eine ausgeweitete Beitragspflicht der Arbeitnehmer mit entsprechenden Leistungsansprüchen abstellt, nicht unberücksichtigt bleiben, wie im SGB III und SGB II dann der Status „arbeitslos“ definiert wird, wenn auch Arbeitslosenversicherungsbeiträge erhoben würden. Bei den Studierenden muss entschieden werden, wie mit dem Status „Werkstudent“ umzugehen ist. Bei den Rentnern steht die Frage an, ob es sinnvoll ist, wenn ein arbeitnehmerbeitragspflichtiger Zuverdienst noch zur Aufstockung von Entgeltpunkten dient. Und bei der Krankenversicherung schließlich stellt sich das Problem, wie sich vermeiden lässt, dass mit einem Mini-Beitrag volle Leistungsansprüche erworben werden.

Doch trotz aller Vielschichtigkeit: Einheitlich dürfte der Widerstand aller Gruppen gegenüber einer vollen Arbeitnehmerbeitragspflicht (auch oberhalb einer Bagatellgrenze) ausfallen, da dies - zumindest im ersten Schritt - zur Verminderung der Nettoeinkommen führt. Das Prinzip brutto gleich netto wird als „Privileg der kleinen Leute“ begriffen und im Einklang mit der Interessenlage der Arbeitgeber verteidigt. $\mathrm{Zu}$ erinnern ist daran, mit welchen Widerstän-

(4) In bis zu 26 Wochen innerhalb der letzten zwölf Monate darf die regelmäßige Arbeitszeit überschritten werden, soweit die Beschäftigung auf die vorlesungsfreie Zeit beschränkt ist oder die Beschäftigung während der Vorlesungszeit zwei Monate nicht übersteigt.

(15) Übersteigen die Einkommen allerdings die Grenzen der Familienversicherung, entfällt der beitragsfreie Krankenversicherungsschutz, der bei einem Studium bis zum 25. Lebensjahr reicht. Auch der Kindergeldanspruch kann dann entfallen. 
den die rot-grüne Regierung im Jahr 1998 konfrontiert war, als damals die Einkommen aus einer geringfügigen Nebenbeschäftigung mit dem Hauptbeschäftigungseinkommen zusammengerechnet und damit voll der Beitrags- und Steuerpflicht unterworfen wurden. Zumindest dieser Schritt, der mit der Reform von 2003 wieder rückgängig gemacht wurde, müsste aber angesichts der Expansion dieser Beschäftigungsform und der damit verbundenen Probleme erneut eingeschlagen werden.

Grundlegendes Ziel einer Neuregelung der geringfügigen Neben- und auch Hauptbeschäftigung ist dabei nicht in erster Linie der Ausbau der eigenständigen Alterssicherung und die Erhöhung von Beitragseinnahmen. Entscheidend wird sein, ob es gelingt, den durch mehrfache Benachteiligungen geprägten Sondercharakter dieses Arbeitsmarktsegments der ge ringfügigen Beschäftigung in den regulären Ordnungsrahmen auf dem Arbeitsmarkt zurückzuholen und die Verzerrungen auf der Angebots- wie auf der Nachfrageseite des Arbeitsmarktes aufzuheben.

Die von der Bundesregierung vorgesehene Anhebung der Geringfügigkeitsgrenze auf $450 €$ und die damit verbundene Verschiebung der Gleitzone auf den Korridor zwischen $450 €$ und $850 €$ steht dieser Zielsetzung entgegen. Da die Anreize, die Erwerbstätigkeit auf ein niedriges Einkommens- und Stundensegment zu begrenzen, verlängert werden, werden die mit den Minijobs einhergehenden Probleme nicht gelöst, sondern fort- und festgeschrieben. Vorgesehen ist auch, dass die bislang freiwillige Rentenaufstockung zur Regel gemacht werden soll und die Beschäftigten nunmehr explizit widersprechen müssen („opting-out“), wenn sie die arbeitnehmerseitigen Rentenbeiträge von 4,6 \% nicht zahlen wollen. Diese Umkehr des Verfahrens mag dazu führen, dass tatsächlich ein wachsender Teil der geringfügig Beschäftigten einen vollwertigen Rentenanspruch erwirbt. Wie gezeigt, bleiben die Rentenansprüche aber äußerst gering. Eine eigenständige Alterssicherung lässt sich nur erreichen, wenn die Arbeitszeit ausgedehnt und das Monatseinkommen deutlich oberhalb der Mini- und Midijob-Grenzen liegt.

\section{LITERATUR}

Bäcker, G. (2007): Was heißt hier "geringfügig“?, in: Keller, B./Seifert, H. (Hrsg.): Atypische Beschäftigung - Flexibilisierung und soziale Risiken, Berlin, S. 107-126

Bäcker, G. (2011): Strategien gegen Armut im Alter in Deutschland, in: Leisering, L. (Hrsg.): Die Alten der Welt, Frankfurt a. M., S. 163-195 Bundesagentur für Arbeit (BA) (2007): Arbeitsmarkt in Zahlen - Beschäftigungsstatistik. Beschäftigung Ende 2006 in Deutschland, Nürnberg Bundesagentur für Arbeit (BA) (2010): Der Arbeitsmarkt in Deutschland: Alleinerziehende im SGB II, Nürnberg

Bundesagentur für Arbeit (BA) (2011a): Qualitätsbericht - Statistik der sozialversicherungspflichtigen und geringfügig Beschäftigten, Version 6.0, Nürnberg
Bundesagentur für Arbeit (BA) (2011b): Arbeitsmarkt in Zahlen - Beschäftigungsstatistik. Beschäftigung in Deutschland, Stichtag 31. Dezember 2010, Nürnberg

Bundesagentur für Arbeit (BA) (2011c): Erwerbstätigkeit von erwerbstätigen Arbeitslosengeld-II-Beziehern, Nürnberg

Deutsche Rentenversicherung (2010): Gleitzonenrechner: www.deutscherentenversicherung.de/cae/servlet/contentblob/32556/publicationFile/19417/ gleitzonenrechner.xls

Deutsches Studentenwerk (2010): Die wirtschaftliche und soziale Lage der Studierenden in der Bundesrepublik Deutschland 2009 - 19. Sozialerhebung, Berlin

Dietz, M./Müller, G./Trappmann, M. (2009): Warum Aufstocker trotz Arbeit bedürftig bleiben, in: IAB-Kurzbericht (2), Nürnberg

Koch, A./Bäcker, G. (2003): Mit Mini- und Midi-Jobs aus der Arbeitslosigkeit?, in: Sozialer Fortschritt 52 (4), S. 94-102

Körner, Th./Puch, K. (2009): Der Mikrozensus im Kontext anderer Arbeitsmarktstatistiken, in: Wirtschaft und Statistik (6), S. 528-552

Minijob-Zentrale (2010): Aktuelle Entwicklungen im Bereich der geringfügigen Beschäftigung, IV. Quartal 2010, Bochum

Minijob-Zentrale (2011): Aktuelle Entwicklungen im Bereich der geringfügigen Beschäftigung, II. Quartal 2011, Bochum

Rheinisch-Westfälisches Institut für Wirtschaftsforschung (RWI) (2004):

Aspekte der Entwicklung von Minijobs, Essen

Rheinisch-Westfälisches Institut für Wirtschaftsforschung (RWI)/Institut für Sozialforschung und Gesellschaftspolitik (ISG) (2006): Evaluation der Umsetzung der Vorschläge der Hartz-Kommission. Verbesserung der beschäftigungspolitischen Rahmenbedingungen und Makrowirkungen der aktiven Arbeitsmarktpolitik, Manuskript

Statistisches Bundesamt (2011a): Verdienste und Arbeitskosten - Arbeitnehmerverdienste, Fachserie 13, Reihe 2.3, Wiesbaden

Statistisches Bundesamt (2011b): Bildung und Kultur - Studierende an den Hochschulen, Fachserie 11, Reihe 4.1, Wiesbaden

Statistisches Bundesamt (2011c): Bildung und Kultur - Allgemeinbildende Schulen, Fachserie 11, Reihe 1, Wiesbaden

Wirtschafts- und Sozialwissenschaftliches Institut (WSI) (2011): Statistisches Taschenbuch Tarifpolitik 2011, Düsseldorf

\section{AUTOREN}

GERHARD BÄCKER, Prof. Dr., lehrt am Institut für Soziologie der Universität Duisburg-Essen. Arbeitsschwerpunkte: Soziologie des Sozialstaates, insbesondere Alterssicherung, Arbeitsmarkt, Armut.

gerhard.baecker@uni-due.de

STEFANIE NEUFFER, Dipl. Soz.-Wiss, ist wissenschaftliche Mitarbeiterin am Institut Arbeit und Qualifikation (IAQ) der Universität Duisburg-Essen sowie am Lehrstuhl für Soziologie des Sozialstaats des Instituts für Soziologie der Universität Duisburg-Essen. Arbeitsschwerpunkte: Sozialpolitik, Arbeitsmarktsoziologie und -politik.

stefanie.neuffer@stud.uni-due.de 